\title{
Case Report: Kanamycin Ototoxicity and MDR-TB Treatment Regimen
}

\author{
Bahru Mantefardo' \\ Gizaw Sisay $\mathbb{D}^{2}$ \\ 'Department of Internal Medicine, \\ School of Medicine, College of Medicine \\ and Health Sciences, Dilla University, \\ Dilla, Ethiopia; ${ }^{2}$ School of Public Health, \\ College of Medicine and Health Sciences, \\ Dilla University, Dilla, Ethiopia
}

\begin{abstract}
Introduction: Aminoglycosides are ototoxic drugs because they have the ability to destroy the inner ear structures irreversibly. They are used to treat Gram-negative bacterial infections that are aerobic and as a second-line treatment for tuberculosis.

Case Presentation: A 40-year-old male from Dilla presented with right side chest pain and cough which is productive of whitish sputum of one-year duration, after investigation the diagnosis of multiple-drug resistant tuberculosis (MDR-TB) was made and the patient was started with a short-term MDR-TB treatment regimen (4-6 KM-Mf-Pto-Cfz-Z-HH-E/5MfxCfz-Z-E). Two and half months after the initiation of treatment, he developed decreased bilateral hearing ability and he had also vertigo, but this patient has no hearing impairment before the initiation of the anti-TB treatment. Then the diagnosis of sensor neural hearing loss secondary to drug toxicity (kanamycin) was made. Then the treatment was discontinued for four days as a result of ototoxicity and the patient was referred to Yirgalem Hospital for further workup and management.
\end{abstract}

Conclusion: Injectable-containing MDR-TB regimens can cause permanent hearing loss. Hearing loss during treatment for MDR-TB with kanamycin can occur at any time. Systematic monitoring of AEs during and after the end of treatment needs to be strengthened in most TB programs. It is important to monitor for hearing loss and kidney function.

Keywords: multiple-drug resistant tuberculosis, aminoglycosides, ototoxicity, Ethiopia, MDRT

\section{Introduction}

Multiple-drug resistant tuberculosis (MDR-TB), tuberculosis resistant to at least rifampicin (RMP) and isoniazid (INH), is a growing and serious threat to tuberculosis control.

The most commonly proposed treatment regimens are long, complex, poorly tolerated, expensive, and only moderately effective, making it difficult to choose an effective, efficient, and appropriate treatment regimen. Treatment for MDR-TB requires an aminoglycoside like kanamycin, amikacin, or capreomycin for eight months or longer, or a shorter course of four months or more, depending on the resistance of the patient's Mycobacterium tuberculosis isolate to other drugs in the regimen. ${ }^{1}$

Aminoglycosides may cause serious side effects, such as permanent ototoxicity, which is estimated to occur in $25-60 \%$ of MDR-TB patients, depending on measurement methods. Even if a patient survives to heal, MDR-TB care is financially draining, and ototoxicity makes it even more difficult to reclaim jobs and can exacerbate social isolation. ${ }^{2}$

Aminoglycoside-induced hearing loss is linked to the selective destruction of the ciliated cells of the cochlea, which begins with those that detect high frequency
Correspondence: Gizaw Sisay

Tel +251911580679

Email gizsisay@gmail.com 
noises and progressively progresses to those that detect low frequency sounds. As a result, unless routine audiometry tests are performed, neither the patient nor his or her doctor will be able to detect the damage at first. ${ }^{3}$

A Comparative retrospective cohort done in Namibian showed hearing loss to be sensorineural, predominantly bilateral, and started with higher frequencies before moving to lower conversational-level frequencies as the severity of deafness grew. The pathogenesis of aminoglycosideinduced hearing loss is consistent with this finding. Aminoglycosides penetrate the inner ear fluids of the organ of Corti and sensory hair cells after parenteral injection, where they are expected to combine with heavy metal ions to create highly reactive free radicals that destroy the sensory hair cells' stereocilia. ${ }^{4}$

With better adherence to WHO guidelines on DR-TB therapy, the incidence of ototoxic hearing loss is projected to decrease (i.e., decreased use of aminoglycosides, particularly kanamycin). While some statistics (e.g., use of alloral DRTB regimens, bedaquiline importation) reveal trends of successful adherence to WHO guidelines, the 2019 data of oral regimens reported in this study suggests that there is still room for improvement. ${ }^{5}$

Drug-resistant tuberculosis (DR-TB) strains are more difficult to treat than drug-susceptible strains, presenting a challenge to global progress toward the World Health Organization's End TB Strategy's goals (WHO). As a result, evidence-based policy guidelines on the treatment and care of patients with DR-TB are urgently needed, based on the most current and thorough evidence available. The WHO unified recommendations on DR-TB treatment fulfill WHO's mission to educate health practitioners in Member States on how to improve treatment and care for DR-TB patients. ${ }^{6}$

A specific type of DR-TB is an MDRT. It means that Mycobacterium tuberculosis, the tuberculosis causative agent, is resistant to two of the most effective antituberculosis drugs, INH and RMP. This indicates that the individual is immune to the above medication and may need a different regimen designed specifically for MDR-TB patients, which is divided into an intensive and a continuous phase. $^{7}$

\section{Case Presentation}

A 40-year-old male from Dilla presented with right side chest pain and cough which is productive of whitish sputum of one-year duration. These symptoms were worsened in the last three months the patient has also night sweating, fever, loss of appetite, and loss of weight but he has no previous history of TB treatment and contact to the TB patient.

\section{On Physical Examination}

He was chronically sick-looking. V/S: BP-100/70, PR120, RR-24, T-37.2̊ C. He has pink conjunctivae and a nonicteric sclera.

- CHEST - there is dullness and decreased air entry on the right posterior lower $2 / 3$ of the lung field. CVSS1 and S2 are well heard.

- Abdomen-flat and soft to palpation. CNS-conscious and oriented to time, place, and person (TPP).

- The neurologic exam findings before the initiation of treatment were normal.

\section{The Diagnosis of Disseminated MDR-TB} Was Made with Laboratory Results Listed as Follows

ESR $-104 \mathrm{~mm} / \mathrm{hr}, \mathrm{WBC}=1,346,000, \mathrm{HCT}=33 \%$, PLT $=$ 425,000, HIV test $=$ NR. Gene Xpert Mycobacterium tuberculosis was detected with RMP resistance. Chest-X-ray showed right side homogeneous opacity with mass effect, with the index of massive pleural effusion. MDR-TB treatment was started with a short-term regimen (4-6 KM-MfPto-Cfz-Z-HH-E/5Mfx-Cfz-Z-E). Two and half months after the initiation of treatment he developed decreased hearing ability which is bilateral and he had also vertigo. Then the treatment was discontinued for 4 days.

\section{Neurologic Examination}

The patient was conscious and oriented to time, place, and person. There is decreased hearing ability bilaterally with sensorineural hearing loss and gait abnormality. The diagnosis of sensorineural hearing loss secondary to drug toxicity (kanamycin) was made and the patient was referred to Yirgalem Hospital for further workup and management.

\section{Discussion}

Treatment of MDR-TB is more complex than treatment of drug-susceptible TB due to the long duration of treatment and serious drug side effects which can be reversible or permanent. These side effects are preventive and also reversible if they are identified and treated early.

Aminoglycosides have a narrow therapeutic index; hence require careful monitoring of serum levels, particularly during their prolonged use in MDR-TB treatment, to 
prevent the occurrence of dose-dependent ototoxicity. In addition, regular audiological assessments may help in the early detection of hearing impairment, before the damage becomes extensive and irreversible.

Hearing loss during treatment for MDR-TB with kanamycin can occur at any time but the severity depends on the duration of treatment and the dose. Kanamycin is concentration-dependent inactivity. Injectable-containing MDR-TB regimens can cause permanent hearing loss. Systematic monitoring of AEs during and after the end of treatment needs to be strengthened in most TB programs. It is important to monitor for hearing loss and kidney function. Permanent ototoxicity with these drugs is an earnest issue and needs meticulous monitoring.

\section{Conclusion}

Injectable-containing MDR-TB regimens can cause permanent hearing loss. Systematic monitoring of adverse events (AEs) during and after the end of treatment needs to be strengthened in most TB programs. It is important to monitor for hearing loss and kidney function. Hearing loss during treatment for MDR-TB with kanamycin can occur at any time. And we want to conclude that these aminoglycosides can cause severe side effects.

\section{Abbreviations}

ADR, Adverse drug reaction; AEs, Adverse events; MDR-TB, Multi-drug resistant tuberculosis; WHO, World Health Organization; XDR-TB, Extensively drug resistant tuberculosis.

\section{Ethics Approval}

The ethical letter was obtained from the Ethical Review Committee of Dilla University, College of Medicine and Health science with protocol unique number $002 / 20-08$ ) to conduct the case. The study purpose, procedures, possible risks, and benefits were explained to participants in local languages. Informed written consent was obtained and signed.

\section{Consent to Publication}

Informed consent was obtained from the patient to publish the case details.

\section{Disclosure}

The authors declare no potential conflicts of interest in this work.

\section{References}

1. Kuaban C, Noeske J, Rieder HL, Aït-Khaled N, Abena Foe J, Trébucq A. High effectiveness of a 12-month regimen for MDR-TB patients in Cameroon. Int J Tuberc Lung Dis. 2015;19(5):517-524. doi:10.5588/ijtld.14.0535

2. Heysell SK, Ahmed S, Rahman MT, et al.. Hearing loss with kanamycin treatment for multidrug-resistant tuberculosis in Bangladesh. Eur Respir J. 2018;51(3):1701778.

3. Isabel JGA, Miroslava FP, Rafael LL. Second-line injectable drugs for the treatment of multidrug-resistant tuberculosis. Why do we keep using them? Available from: http://www.ramr.org/articulos/volumen 19 numero 3/articulos originales/articulos_originales second line injectable_drugs_for_the_treatment_of_multidrug-resistant_tuberculo sis_why_do_we_keep_using_them.pdf. Accessed November 22, 2021.

4. Sagwa EL, Ruswa N, Mavhunga F, Rennie T, Leufkens HG, MantelTeeuwisse AK. Comparing amikacin and kanamycin-induced hearing loss in multidrug-resistant tuberculosis treatment under programmatic conditions in a Namibian retrospective cohort. BMC Pharmacol Toxicol. 2015;16(1). doi:10.1186/s40360-015-0036-7

5. Dillard LK, Martinez RX, Perez LL, Fullerton AM, Chadha S, McMahon CM. Prevalence of aminoglycoside-induced hearing loss in drug-resistant tuberculosis patients: a systematic review: ototoxic hearing loss in drug-resistant tuberculosis. J Infect. 2021;83:27-36.

6. World Health Organization. WHO consolidated guidelines on drug-resistant tuberculosis treatment. World Health Organization; 2019.

7. Baqar T, Ahmad S, Rai S. Ototoxicity in MDR-TB patients on category-4 regimen: a case report. Era's J Med Res. 2020;7 (2):257-259. doi:10.24041/ejmr2020.45

\section{Publish your work in this journal}

The International Medical Case Reports Journal is an international, peer-reviewed open-access journal publishing original case reports from all medical specialties. Previously unpublished medical posters are also accepted relating to any area of clinical or preclinical science. Submissions should not normally exceed 2,000 words or 4 published pages including figures, diagrams and references. The manuscript management system is completely online and includes a very quick and fair peer-review system, which is all easy to use. Visit http://www.dovepress.com/testimonials.php to read real quotes from published authors. 